\title{
CHARACTERIZING $\Omega$-STABILITY FOR FLOWS IN THE PLANE
}

\author{
MARIA LÚCIA ALVARENGA PEIXOTO
}

(Communicated by Kenneth R. Meyer)

\begin{abstract}
In this paper, the $C^{r} \Omega$-stability for flows in the plane is characterized using the notion called "generalized recurrence" by J. Auslander [1].
\end{abstract}

The $\Omega$-stability theory of vector fields on noncompact manifolds is not a simple analogue of the compact theory. The requirement that the equivalence homeomorphism, in the definition of $\Omega$-stability, be near the identity map in the compact-open $C^{0}$ topology is essential, as it is in the definition of the global $C^{r}$ structural stability of vector fields on noncompact surfaces in [3]. Nitecki proves there that on the plane without such requirement, structural stability does not necessarily imply that singularities be hyperbolic. The same is true for $\Omega$-stability. He uses the idea of a composed focus of Sotomayor [7], that is, a singularity which is topologically a sink or a source, but it is not hyperbolic because the eigenvalues of the linearization are pure imaginary.

Let $\mathscr{X}_{0}^{r}(M)$ be the space of all $C^{r}$ vector fields on a noncompact manifold $M$, with the $C^{r}$ Whitney topology [6]. The vector fields which generate flows form an open subspace $\mathscr{X}^{r}(M) \subset \mathscr{X}_{0}^{r}(M)$. The vector field $X \in \mathscr{X}^{r}(M)$ determines a unique flow $\varphi: R \times M \rightarrow M$. For basic notions and facts about $\Omega$-stability see [4].

Nitecki's statement is

1. LEMMA. There exist $C^{r}$ flows $\varphi$ on $\mathbf{R}^{2}(r \geq 4)$ which possess a composed focus and such that $\varphi$ is topologically equivalent to any flow $\psi C^{r}$-near $\varphi$.

The phrase portrait for $\varphi$ is in Figure 1. The strips that contain the discs $D_{1}$ and $D_{-1}$ are translated respectively to the right and to the left indefinitely. According to Nitecki, considering a flow $\psi, C^{4}$-near $\varphi$ it is easy to see that the orbit structure of $\psi$ outside the disc $D_{0}$ is equivalence to that of $\varphi$. Inside, $\psi / D_{0}$ is equivalent either to $\varphi / D_{0}$ or to $\varphi / D_{1}$. In the first case the equivalence takes the disc $D_{n}$ to itself and in the second case, the equivalence takes the $\psi$-portrait in $D_{n}$ to the $\varphi$-portrait in $D_{n+1}$. The equivalence in the second case is not near the identity, but $\varphi$ and $\psi$ are globally equivalent in both cases. For more details see [3]. Thus, the following definition of $\Omega$-stability is the most appropriate for the noncompact manifolds $M$.

2. DEFinition. $X \in \mathscr{X}^{r}(M)$ is said to be $C^{r} \Omega$-stable if for every compact $K \subset M$ and $\varepsilon>0$ there exists a neighborhood $\mathscr{U}$ of $X$ in the $C^{r}$ Whitney topology,

Received by the editors September 24, 1987 and, in revised form, December 1, 1987.

1980 Mathematics Subject Classification (1985 Revision). Primary 58F10.

Key words and phrases. Flows, $\Omega$-stability, generalized recurrence, prolongational limit sets, closing lemma. 


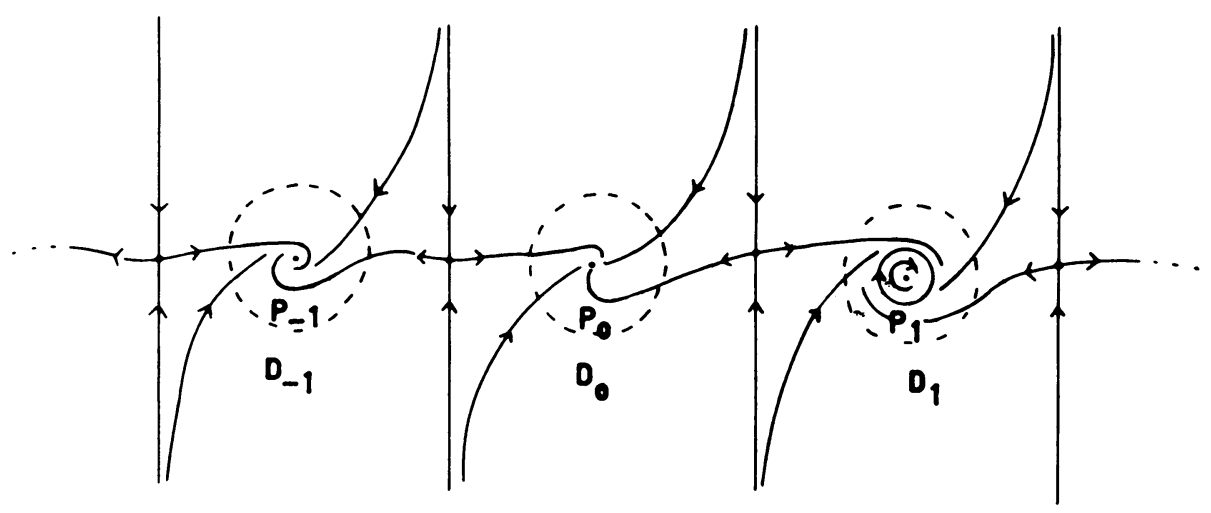

FIGURE 1

$r \geq 1$, such that for each $Y \in \mathscr{U}$ there exists a homeomorphism $h_{Y}: \Omega(X) \rightarrow \Omega(Y)$ which is $\varepsilon$-near the identity in $K$ and takes trajectories of $X$ into trajectories of $Y$.

We can characterize the $\Omega$-stability for flows in the plane using the generalized recurrent set $R(X)$, defined by J. Auslander [1]. Denoting by $\sigma_{i}^{X}$ the critical elements (singularities and closed orbits) of a vector field $X$, we state a theorem for the characterization of the $\Omega$-stability as follows:

3. THEOREM. $X \in \mathscr{X}^{r}\left(\mathbf{R}^{2}\right), r \geq 1$ with all critical elements $\sigma_{i}^{X}$ hyperbolic is $\Omega$-stable if and only if $R(X)=\bigcup_{i} \sigma_{i}^{X}$.

We now define $R(X)$ and for this we need a few other definitions.

4. DEFinition. For each $X \in \mathscr{Q}^{r}\left(\mathbf{R}^{2}\right)$ and $x \in \mathbf{R}^{2}$ we define the first prolongational limit set by

$$
J^{+}(x)=\left\{y \in \mathbf{R}^{2}: \exists x_{n} \rightarrow x, t_{n} \rightarrow \infty \ni \varphi_{t_{n}}\left(x_{n}\right) \rightarrow y\right\} .
$$

5. Definition. For a subset $S \subset \mathbf{R}^{2}$, we define

$$
J^{+}(S)=\bigcup_{x \in S} J^{+}(x) \text {. }
$$

6. Definition. For each ordinal number $\alpha, X \in \mathscr{X}^{r}\left(\mathbf{R}^{2}\right)$ and $x \in \mathbf{R}^{2}$ we call $J_{\alpha}(x)$ the prolongational limit set of order $\alpha$, defined by transfinite induction as follows:

(1) $J_{1}(x)=J^{+}(x)$.

(2) Suppose that for all $\beta<\alpha, J_{\beta}(x)$ is defined.

(i) if $\alpha$ is a successor ordinal number, we set $J_{\alpha}(x)=J_{1}\left(J_{\alpha-1}(x)\right)$;

(ii) if $\alpha$ is a limit ordinal number, we set

$J_{\alpha}(x)=\left\{y \in \mathbf{R}^{2}: \exists x_{n} \rightarrow x, y_{n} \rightarrow y\right.$ and ordinals $\beta_{n}<\alpha$ with $\left.y_{n} \in J_{\beta_{n}}\left(x_{n}\right)\right\}$.

7. DEFINITION. The generalized or prolongational recurrent set $R(X)$ of $X$, also called the Auslander recurrent set, is defined by

$$
R(X)=\left\{x \in \mathbf{R}^{2}: x \in J_{\alpha}(x) \text { for some ordinal number } \alpha\right\} .
$$

8. Definition. A point $p \in R(X)$ which is not a periodic point is called prolongationally recurrent point. 
We use here a weaker version of the Closing Lemma for the generalized recurrent set in [5]:

9. Closing Lemma FOR $R(X)$. Suppose $X \in \mathscr{X}^{r}\left(\mathbf{R}^{2}\right)$ has only hyperbolic singularities. Given $p \in R(X)$, a prolongationally recurrent point, and a neighborhood $\mathscr{U}$ of $X$ in $\mathscr{X}^{r}\left(\mathbf{R}^{2}\right)$ then there exists $Y \in \mathscr{U}$ with a closed orbit through $p$.

We prove the necessary condition of Theorem 3 in the following.

10. Proposition. If $X \in \mathscr{X}^{r}\left(\mathbf{R}^{2}\right)$ is $\Omega$-stable, then $R(X)=\Omega(X)=\bigcup_{i} \sigma_{i}^{X}$.

Proof. Suppose $R(X) \neq \bigcup_{i} \sigma_{i}^{X}$. Consider $p \in R(X)-\bigcup_{i} \sigma_{i}^{X}$. Choose $\varepsilon>0$ and $K$ a compact disc with radius greater than $\varepsilon$ and center in $p$ and such that no closed orbit of $X$ intersects $K$. Since $X$ is $\Omega$-stable, there exists a neighborhood $\mathscr{U}$ of $X$ such that for any $Y \in \mathscr{U}$ there exists a homeomorphism $h_{Y}$ between $\Omega(X)$ and $\Omega(Y)$, which is a $\varepsilon$-homeomorphism on $K$. By the Closing Lemma for $R(X)$, there exists a vector field $Y \in \mathscr{U}$, which has a closed orbit $\gamma_{Y}$ through $p$. Since $\gamma_{Y}$ crosses $K$, by the $\varepsilon$-homeomorphism $h_{Y}$ in $K, X$ has a closed orbit $\gamma_{X}, \varepsilon$-near $\gamma_{Y}$, crossing $K$. This is a contradiction. Then $R(X)=\bigcup_{i} \sigma_{i}^{X}$. Since $\bigcup_{i} \sigma_{i}^{X} \subset \Omega(X) \subset R(X)$, we have $R(X)=\Omega(X)=\bigcup_{i} \sigma_{i}^{X}$.

The condition $R(x)=\bigcup_{i} \sigma_{i}^{X}$ is also a sufficient condition for $\Omega$-stability for vector fields in $\mathbf{R}^{2}$ and this was proved by Fopke Klok in [2]. Although Fopke Klok does not take into account that in the definition of $\Omega$-stability the equivalence homeomorphism should be $C^{0}$-near the identity map on $\mathbf{R}^{2}$ (with respect to the compact open topology), a simple modification of this proof makes it work well for the case when $\Omega$-stability is defined as in (2). This happens because we have a finite number of critical elements of $X$ in each compact $\mathscr{C} \subset \mathbf{R}^{2}$ by the following proposition.

11. PROPOSITION. If $X \in \mathscr{X}^{r}\left(\mathbf{R}^{2}\right)$ has all critical elements $\sigma_{i}^{X}$ hyperbolic and $R(X)=\bigcup_{i} \sigma_{i}^{X}$, then for each compact set $\mathscr{C} \subset \mathbf{R}^{2}$ there is only a finite number of $\sigma_{i}^{X}$ with $\sigma_{i}^{X} \cap \mathscr{C} \neq \varnothing$. For the proof see [2].

This enables us to choose, for vector fields as above, disjoint and sufficiently small neighborhoods of the critical elements $\sigma_{i}^{X}$, in order to prove the existence of a neighborhood $\mathscr{U}(X)$ such that the equivalence homeomorphism between $\Omega(X)$ and $\Omega(Y)$ be $C^{0}$-near the identity in given compacts sets, for $Y \in \mathscr{U}(X)$. Thus the $\Omega$-stability for vector fields in the plane is characterized by Theorem 3 .

\section{REFERENCES}

1. J. Auslander, Generalized recurrence in dynamical systems, Contrib. to Differential Equations 3 (1964), 65-74.

2. F. Klok, $\Omega$-stability of plane vectorfields, Bol. Soc. Brasil. Mat. 12 (1981), 21-27.

3. Z. Nitecki, J. Kotus, and M. Kryck, Global structural stability of flows on open surfaces, Mem. Amer. Math. Soc., no. 261, 1982.

4. J. Palis and W. de Melo, Geometric theory of dynamical systems, Springer-Verlag, Berlin and New York, 1983.

5. M. L. A. Peixoto, The closing lemma for generalized recurrence in the plane, Trans. of Ann. Math. Soc. Vol. 309, number 1, September 1, 1988. 
6. M. M. Peixoto and C. C. Pugh, Structural stability on open manifolds is never dense, Ann. of Math. (2) 87 (1968), 423-430.

7. J. Sotomayor, Generic one-parameter families of vector fields on two-dimensional manifolds, Inst. Hautes Études Sci. Publ. Math. 431974.

ENCE, Rua André Cavalcanti 106, 20231 Rio de Janeiro, R. J., Brasil. 